\title{
Structure des peuplements ligneux des formations végétales de la forêt sacrée de Nassou en zone soudanienne du Bénin
}

\author{
Pierre Onodjè AGBANI ${ }^{1,2}$, Aubin AMAGNIDE ${ }^{3}$, Cédric GOUSSANOU $^{1 *}$, \\ Fortuné AZIHOU ${ }^{1}$ et Brice SINSIN ${ }^{1}$ \\ ${ }^{1}$ Laboratoire d'Ecologie Appliquée, Faculté des Sciences Agronomiques, Université d'Abomey-Calavi, 01 BP \\ 526, Cotonou, Benin. \\ ${ }^{2}$ Centre Béninois de la Recherche Scientifique et de l'Innovation BP 06, Porto-Novo, Bénin. \\ ${ }^{3}$ Laboratoire de Biomathématiques et d'Estimations Forestières, Faculté des Sciences Agronomiques, \\ Université d'Abomey-Calavi, 04 BP 1525, Cotonou, Bénin. \\ *Corresponding author; E-mail: cedricgoussanou@gmail.com
}

\section{RESUME}

Les forêts sacrées connaissent une dégradation proportionnelle à l'augmentation des populations riveraines et du développement de leurs activités socioéconomiques. La présente étude avait pour objectif d'étudier la structure forestière de la forêt sacrée de Nassou, et d'évaluer les possibles effets anthropiques sur les caractéristiques dendrométriques et écologiques de 4 essences fruitières ligneuses. A cet effet, un inventaire forestier de 60 placettes carrées de $900 \mathrm{~m}^{2}$ réparties suivant un échantillonnage stratifié aléatoire dans les trois formations végétales de ladite forêt a été réalisé. De plus, les niveaux de pressions anthropiques ont été notés en suivant une grille d'évaluation. Certains paramètres structuraux ont été calculés et analysés. Les paramètres dendrométriques et écologiques obtenues variaient globalement en fonction des formations végétales pour les quatre espèces. En général, les plus fortes valeurs sont obtenues en forêt dense sèche par comparaison aux autres formations. Les distributions en diamètre au sein de la forêt présentent globalement des allures normales mais la structure de chaque peuplement d'espèce présente des allures diverses. De plus, une corrélation négative et non significative de manière globale a été observée entre les niveaux de pression et les espèces cibles, indiquant une pression anthropique dirigée vers d'autres espèces. Il faudra donc continuer les investigations pour identifier les espèces les plus vulnérables au choix sélectif des populations et leur effet sur la viabilité des peuplements.

(C) 2018 International Formulae Group. All rights reserved.

Mots-clés: Structure, régénération, pressions anthropiques, Forêt sacrée, Nassou.

\section{Ligneous stand structure of vegetation types of sacred Forest of Nassou in Sudanian zone of Benin}

\begin{abstract}
Sacred forests are deteriorating in proportion to the increase in riparian populations and the development of their socio-economic activities. This study assessed forest structure of sacred grove Forest of Nassou and possible human effect on biometric and ecological parameters of four fruit plant species. To that
\end{abstract}


end, a forest inventory in 60 plots of $900 \mathrm{~m}^{2}$ were established using a stratified random across the three vegetation types of the forest. In addition, pressure scale were used to appreciate pressure threshold on target species. Some structural parameter were calculated and statistically tested. Main results shown a variation of biometric and ecological following vegetation types with the highest values obtained in dry forest. Diameter structure were globally followed normal distribution for the whole forest, whereas per species and across vegetation the distribution varies a lot. Moreover, negative correlation between human pressure and diameter structure of the target species. This indicate a pressure on others species of the forest leading to further studies to identify vulnerable species to selective choice by local population and consequences on their viability.

(c) 2018 International Formulae Group. All rights reserved.

Keywords: Structure, regeneration, human pressure, Sacred forest, Nassou.

\section{INTRODUCTION}

Les forêts sacrées sont retrouvées un peu partout dans la zone tropicale et sont le siège de rites cultuels, culturels et religieux qui ont permis de préserver la biodiversité à travers la conservation communautaire (Savadogo et al., 2017). Ces forêts sacrées, refuges pour des espèces endémiques, participent à la conservation de la biodiversité, fournissent de nombreux biens et services écosystémiques et contribuent aux objectifs internationaux de conservation (Dudley et al., 2009 ; Udeagha et al., 2013 ; Tamalane et al., 2014). On note un regain d'intérêt par les scientifiques et les organismes internationaux pour les forêts sacrées dans toute la zone tropicale (Ormsby et Bhagwat, 2010 ; Cardelús et al., 2013; Gutiérrez, 2014 ; Brandt et al., 2015).

Dans la zone savanicole ouestafricaine, en dehors des aires protégées, les îlots forestiers sont presque tous des forêts sacrées (Juhé-Beaulaton, 2010). Au Bénin, la végétation forestière est limitée à environ 1000 forêts sacrées, la plupart couvrant moins d'un (1) ha de superficie (Neuenschwander et al., 2015). Diverses études ont porté sur les forêts sacrées traitant de leurs rôles, leur conservation et leur diversité biologique (Ali et al., 2014; Alohou et al., 2016. ;Yedomonhan et al., 2017). Au Bénin, la préservation des forêts rémanentes notamment celles dite sacrées est considérée comme prioritaire (Neuenschwander et al., 2011). De nos jours, en dépit de leur importante valeur au plan écologique et socioculturel, les forêts sacrées sont dégradées, réduites en superficie et tendent à disparaître, dû à l'expansion démographique croissante, la recherche de terre cultivables fertiles, l'influence des religions importées et la perte de pouvoir des autorités coutumières traditionnelles (Kokou et Sokpon, 2006 ; Ali et al., 2014).

A l'échelle nationale, les différentes études sur les forêts sacrées sont localisées au Sud-Bénin et portent essentiellement sur leurs fonctions socio-culturelles (Kokou et Sokpon, 2006; Alohou et al., 2016). Les différentes menaces telles que la culture extensive de l'igname, le coton et l'exploitation illégale et parfois sélective du bois affectent la structure forestière et la biodiversité des forêts sacrées (Siebert et Elwert, 2004; Ali et al., 2014) et particulièrement celle du Nord-Bénin telle que la forêt sacrée de Nassou. L'exploitation forestière très sélective, incontrôlée constitue une menace pour certaines espèces (Agbessi et al., 2017). Des espèces comme Khaya senegalensis, Afzelia africana, Milicia excelsa, Antiaris toxicaria, Pterocarpus erinaceus, Afraegle paniculata, Kigelia africana, Diospyros mespiliformis et Vitex doniana se raréfient quotidiennement et apparaissent fortement menacées (Neuenschwander et al., 2011). Ces actions anthropiques ont considérablement réduit les effectifs de certaines espèces utiles présentes dans la forêt sacrée de Nassou et des espaces avoisinants. Dans une moindre mesure, beaucoup d'autres espèces bien que moins convoitées pour l'ébénisterie mais très recherchées à des fins énergétiques ou autres sont aussi menacées de survie à court et à moyen terme avec la forte pression anthropique. Des études approfondies sont 
donc nécessaires pour apprécier la diversité biologique et l'effet des perturbations humaines sur certaines espèces de valeur dans des écosystèmes ne disposant d'aucunes données de références parfois. L'objectif de cette étude sont (i) de décrire la structure de certaines espèces menacées de la forêt, (ii) et d'évaluer les probables effets anthropiques sur la viabilité du peuplement ligneux afin de fournir des éléments de décision pour sa gestion durable.

\section{MATERIEL ET METHODES Milieu d'étude}

L'étude a été réalisée dans la forêt de Nassou, située dans la commune de OuassaPéhunco (Figure 1) en zone soudanienne du Bénin. Située entre la haute vallée de l'Alibori à l'Est et celle du Mékrou à l'Ouest dans le Sud-Est du Département de l'Atacora entre $10^{\circ} 03^{\prime}$ et $10^{\circ} 45^{\prime}$ de latitude Nord et $1^{\circ} 45^{\prime}$ et $2^{\circ} 15^{\prime}$ de longitude Est. La forêt de Nassou est une mosaïque de forêt dense sèche, de savane et de jachères. La gestion de la forêt de Nassou est assurée par l'association communale des Praticiens de la Médecine Traditionnelle appelée en

Baatonou «Faaba Yéru » et son bras armé « Tim Gbéru ». Son sens d'organisation, sa puissance et la satisfaction donnée aux populations locales lui confère une notoriété à tel point qu'elle est très sollicitée pour des questions relatives à la gestion des ressources naturelles locales de la Commune de OuassaPéhunco. Un des membres de cette association spécialiste dans le traitement des maladies démentielles qui habite proche de cette forêt en assure la garde avec ses collègues du village de Nassou qui abrite la forêt.

\section{Méthodologie}

\section{Echantillonnage et collecte de données}

La caractérisation de la végétation de l'aire d'étude a été réalisée au moyen d'un inventaire forestier. Une stratification a priori de la végétation a été effectuée en tenant compte du type de formation végétale. Ainsi, 60 placettes carrées de $900 \mathrm{~m}^{2}$ ont été aléatoirement installées en forêt dense, savane arborée et jachère. Proportionnellement à la superficie de chaque type de formation végétale, 32 placettes ont été installées dans la forêt dense contre 28 équitablement répartis entre la savane arborée et la jachère. Cinq quadrats de $100 \mathrm{~m}^{2}$ ont été établis au centre et aux quatre sommets de chaque placette de 900 $\mathrm{m}^{2}$.

La collecte des données dans les placettes d'inventaire s'est faite au moyen d'un ruban pi pour la mesure du diamètre des arbres, d'un GPS pour l'enregistrement des coordonnées géographiques des placettes d'inventaire et enfin une fiche de collecte de données pour y relever les différentes mensurations. Dans les placettes de $900 \mathrm{~m}^{2}$, tous les arbres de diamètre à $1,30 \mathrm{~m}$ du sol (dbh) supérieur ou égal à $5 \mathrm{~cm}$ ont été dénombrés puis leur diamètre mesuré.

Le niveau de pression sur la végétation y a été également apprécié en suivant une échelle de pression codifié comme suit: 1 pour une faible pression, 2 pour une pression moyenne et 3 pour une forte pression. L'évaluation de la régénération (individus de dbh inférieur à $5 \mathrm{~cm}$ ) a été faite dans les cinq quadrats diagonaux de $100 \mathrm{~m}^{2}$ installés dans les placettes de $900 \mathrm{~m}^{2}$. Les espèces non identifiées sur le terrain ont été herborisées et ramenées à l'herbier national pour identification.

Traitement et analyse statistique des données

Identification des espèces ligneuses à valeur d'importance

Toutes les analyses statistiques ont été réalisées avec le logiciel $\mathrm{R}$ version 3.0.2 ( $\mathrm{R}$ Core Team, 2013) et le seuil de signification a été fixé à 0.05 . Pour identifier les principales espèces ligneuses de chaque formation végétale, l'indice de valeur d'importance (IVI) de chaque espèce (Curtis et Macintosh, 1951) a été calculé par formation végétale. Ainsi, pour une espèce $\alpha$, l'IVI est donné par:

$$
I V I_{\alpha}=R D_{\alpha}+R F_{\alpha}+R C_{\alpha} .
$$

où

$$
R D_{\alpha}=n_{\alpha} / \sum_{i=1}^{k} n_{\alpha}
$$

est la densité relative de l'espèce $\alpha$; 


$$
R F_{\alpha}=f_{\alpha} / \sum_{\alpha=1}^{k} f_{\alpha} ; f_{\alpha}=j_{\alpha} / k
$$

est la fréquence relative de l'espèce $\alpha ; j_{\alpha}$ est le nombre de placettes dans lequel l'espèce a été observée et $k$ le nombre total de placettes.

$$
R C_{\alpha}=C_{\alpha} / \sum_{\alpha=1}^{n} C_{\alpha} ; C_{\alpha}=a_{\alpha} N_{\alpha} / n_{\alpha} \text { (4) }
$$

est la dominance relative de l'espèce $\alpha ; a_{\alpha}$ est la surface terrière de l'espèce $\alpha ; \mathrm{N} \alpha$ est la densité de l'espèce $\alpha$ et n $\alpha$ est le nombre total d'individus échantillonnés pour l'espèce $\alpha$.

L'IVI varie de 0 à 3 et traduit le pourcentage d'importance. Il donne une estimation globale du niveau d'importance d'une plante dans une communauté végétale (Assogbadjo et al., 2009). Plus la valeur de l'IVI d'une espèce est élevée, plus cette espèce est écologiquement importante. Le diagramme en barre des valeurs de l'IVI a été établi pour les cinq espèces les plus écologiquement importantes par formation végétal identifié.

\section{Caractérisation structurale des formations végétales}

Paramètres de diversité floristique

Pour chaque formation végétale, les paramètres de diversité floristique que sont la richesse spécifique, l'indice de diversité de Shannon et l'équitabilité de Piélou ont été calculés. Ces paramètres ont fait l'objet de comparaison entre les formations végétales.

- $\quad$ Richesse spécifique ( $S$ en espèces): elle représente le nombre total d'espèces présentes dans un milieu donné.

- Indice de diversité de Shannon ( $H$ en bits), défini par la formule suivante :

$$
H=-\sum_{i=1}^{s} \frac{n_{i}}{n} \log _{2} \frac{n_{i}}{n}
$$

$n_{i}=$ nombre d'individus de l'espèce $i$ et $n=$ nombre total d'individus inventoriés dans les placettes.

- $\quad$ Equitabilité de Piélou (Eq): elle est une mesure de la stabilité du peuplement et équivaut au rapport de $H$ à l'indice maximal théorique dans le peuplement $\left(H_{\max }\right)$ :

$$
E q=\frac{H}{H_{\text {max }}} \quad \text { avec } \quad H_{\text {max }}=\log _{2} S
$$

Paramètres dendrométriques

Pour chaque formation végétale identifiée, les paramètres dendrométriques que sont la densité d'arbres, le diamètre moyen et la surface terrière (Rondeux, 1999; Kangas et Maltamo, 2007) ont été calculés. Ces paramètres ont été soumis à des analyses de variance (ANOVA) ou à défaut des tests de Kruskall-Wallis (lorsque les conditions d'application de l'ANOVA ne sont pas satisfaites) pour tester si les différences entre les formations végétales sont significatives. De plus, la contribution en surface terrière $(\mathrm{Cs}, \%)$ et la densité de régénération $(\mathrm{Nr}$, plants/ha), ont été aussi calculés et ont fait l'objet de comparaison entre formations végétales identifiés pour quatre espèces ligneuses (A. paniculata, D. mespiliformis, $K$. africana et $V$. doniana.) très usités par les populations locales (Achigan-Dako et al., 2010 ; Agbani et al., 2017).

- Densité $d u$ peuplement $(N \quad$ en arbres/ha): elle est le nombre moyen d'arbres de $\mathrm{dbh} \geq 5 \mathrm{~cm}$ par hectare. Elle est donnée par la formule:

$$
N=\frac{n}{s}
$$

$n$ est le nombre total d'arbres par placeau et $s$, la surface du placeau en ha.

- $\quad$ Diamètre moyen $(D g$ en $\mathrm{cm})$ : c'est le diamètre de l'arbre de surface terrière moyenne. Il est déterminé par:

$$
D g=\sqrt{\frac{1}{n} \sum_{i=1}^{n} d_{i}^{2}}
$$

$n=$ nombre d'arbres du placeau et $d_{i}=$ diamètre $(\mathrm{cm})$ de l'arbre $i$.

- $\quad$ Surface terrière $\left(G\right.$ en $\left.\mathrm{m}^{2} / \mathrm{ha}\right)$ : pour tout le peuplement, elle est la somme de la section transversale à hauteur d'homme $(1,30 \mathrm{~m})$ de tous les arbres de $\mathrm{dbh} \geq 10$ $\mathrm{cm}$, à l'intérieur du placeau. 
$G=\frac{0,0001 \pi}{4 s} \sum_{i=1}^{n} d_{i}^{2}$

$\mathrm{d}_{\mathrm{i}}=$ diamètre en centimètre $(\mathrm{cm}) \mathrm{de}$ l'arbre i du placeau et $\mathrm{s}=$ surface $\mathrm{du}$ placeau en ha.

- Contribution en surface terrière (Cs, en\%): elle est définie comme étant la part de l'espèce dans la surface terrière de l'ensemble des individus de l'unité d'échantillonnage:

$$
C s=100 \frac{G p i}{G}
$$

Gpi étant la surface terrière des individus de l'espèce $i$ et $G$ celle de l'ensemble des individus du placeau.

- Densité de régénération ( $\mathrm{Nr}$, en plants/ha)

$$
N r=\frac{1}{5} \sum_{i=1}^{5} d r_{i} \quad \text { avec } \quad d r_{i}=\frac{n_{i}}{s q}
$$

$d r_{i}$ étant la densité de régénération dans le quadrat $i ; n_{i}$ le nombre de régénération et $s q$ la surface du quadrat.

Pour évaluer l'effet des pressions anthropiques sur la structure de ces espèces, les corrélations de Spearman entre les niveaux de pression dans les unités d'échantillonnage et les paramètres dendrométriques des espèces ont été calculées.

Etablissement des structures en diamètre des arbres

Les structures en diamètre sont révélatrices des évènements liés à la vie des peuplements (Rondeux, 1999). Ainsi, pour chaque formation végétale et chacune des populations des espèces cibles, la structure en diamètre a été établie. La distribution à trois paramètres a (paramètre de seuil), b (paramètre d'échelle) et $c$ (paramètre de forme) de Weibull (Johnson et Kotz, 1970) a été ajustée aux structures observées. Toutefois, les structures de $A$. paniculata et $D$. mespiliformis dans la forêt dense ont été ajustées à la distribution exponentielle à deux paramètres a et $b$, parce que ne s'ajustant pas à la distribution à trois paramètres de Weibull. La distribution de Weibull peut prendre plusieurs formes selon la valeur du paramètre de forme (c):

- $\quad \mathrm{c}<1$ : distribution en « $\mathrm{J}$ renversé », caractéristique des peuplements multispécifiques ou inéquiennes;

- $\quad c=1$ : distribution exponentiellement décroissante, caractéristique des populations en extinction;

- $\quad 1<\mathrm{c}<3,6$ : distribution asymétrique positive ou asymétrique droite, caractéristique des peuplements monospécifiques avec prédominance d'individus jeunes ou de faible diamètre; - $\mathrm{c}=3,6$ : distribution symétrique, structure normale, caractéristique des peuplements équiennes ou monospécifiques de même cohorte;

- $\quad$ c > 3,6: distribution asymétrique négative ou asymétrique gauche, caractéristique des peuplements monospécifiques à prédominance d'individus âgés ou de gros diamètre.

L'analyse log-linéaire a été réalisée pour tester l'adéquation globale entre les distributions observées et celles théoriques. Il a été alors possible à partir de ces paramètres de tirer des conclusions sur l'état de conservation des groupements végétaux en général et en particulier sur celui des populations des espèces cibles suivant les groupements végétaux identifiés. 


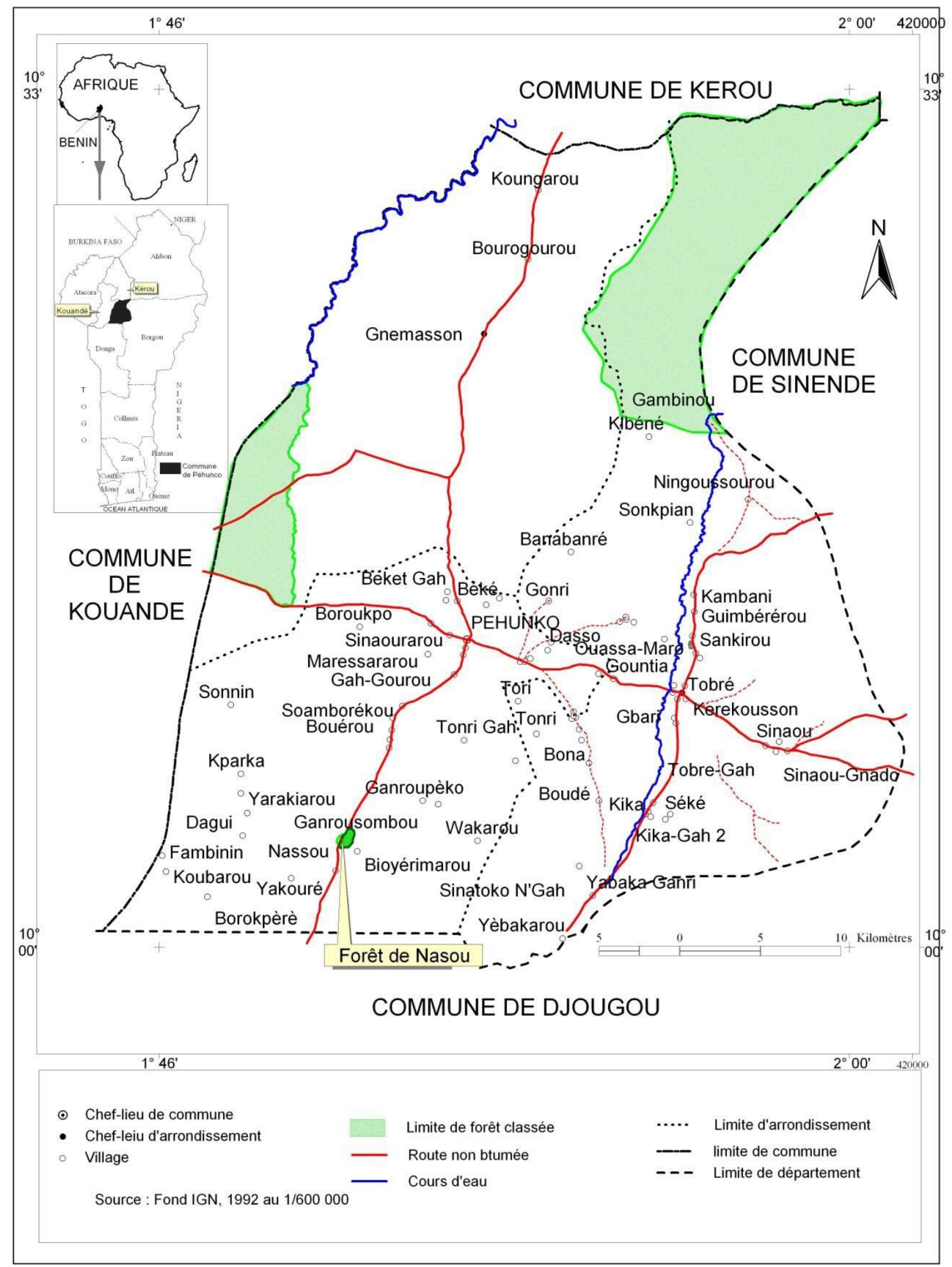

Figure 1 : Localisation de la forêt de Nassou dans la Commune de Ouassa-Péhunco. 
RESULTATS

\section{Caractéristiques structurales des formations végétales Diversité floristique}

La forêt dense avait une richesse spécifique moyenne de 17,94 espèces par placette pour une richesse spécifique globale de 92 espèces (Tableau 1). Les valeurs élevées observées pour la diversité de Shannon et l'équitabilité de Piélou indiquent une bonne diversité de la végétation ligneuse dans cette formation végétale. Les espèces les plus écologiquement importantes en forêt dense (Figure 2) sont Anogeissus leiocarpa (IVI = $0,4159)$ et Zanha golungensis (IVI $=0,1338$ ) alors que la moins importante est Entada abyssinica $(I V I<0,0025)$.

La savane arborée présente une richesse spécifique moyenne de 1,93 espèce par placette pour une richesse spécifique globale de 6 espèces (Tableau 1). L'indice de diversité de Shannon ( $\mathrm{H}=1,55$ bits) et l'Equitabilité de Piélou $(\mathrm{Eq}=0,60)$ sont assez faibles. Les espèces les plus écologiquement importantes de la savane arborée (Figure 2) sont Pterocarpus erinaceus $(I V I=1,4411)$ et A. africana $(I V I=0,5205)$ alors que les moins importantes sont Burkea africana et $D$. mespiliformis (IVI $=0,2058$ et $I V I=0,1032$ respectivement).

En ce qui concerne la jachère, les valeurs faibles des paramètres de diversité floristique observées indiquent que la formation végétale est peu diversifiée en ce qui concerne la végétation ligneuse (Tableau 1). Parkia biglobosa $(I V I=1,0631)$ et $D$. mespiliformis $(I V I=0,8960)$ y sont les espèces les plus écologiquement importantes (Figure 2).

\section{Caractéristiques dendrométriques}

Les résultats de l'analyse de variance (Tableau 2) effectuée sur les caractéristiques dendrométriques des populations des quatre espèces cibles et les populations globales révèlent des différences significatives (Prob. $<0,05)$ entre d'une part la densité d'arbres et d'autre part, la contribution en surface terrière de $V$. doniana; entre d'une part la contribution en surface terrière et d'autre part, la densité de régénération de $D$. mespiliformis et entre la densité d'arbres d'une part et la surface terrière d'autre part des populations globales. L'analyse comparative des caractéristiques dendrométriques des formations végétales (Tableau 2) révèle que la forêt dense présente les valeurs les plus élevées $(N=937,50$ tiges/ha, $D=18,94 \mathrm{~cm}$ et $G=25,82 \mathrm{~m}^{2} / \mathrm{ha}$ ) alors que la jachère en présente les plus faibles valeurs $(N=59,26$ tiges/ha, $D=16,59$ cm et $G=1,55 \mathrm{~m}^{2} / \mathrm{ha}$ ). En ce qui concerne les espèces cibles, A. paniculata et $K$. africana, seulement observées en forêt dense avaient des densités moyennes de 2,43 et 20,14 tiges/ha (respectivement) pour des diamètres moyens de 52,78 et $26,54 \mathrm{~cm}$ (respectivement), des surfaces terrières de 8,35 et $2,31 \mathrm{~m}^{2} / \mathrm{ha}$ (respectivement), des contributions en surface terrière de 26,38 et $7,92 \%$ (respectivement) et des densités de régénération de 20,00 et 133,33 plants/ha (respectivement). Toutefois, des régénérations de $K$. africana étaient observées en jachère (260,00 plants/ha; Tableau 2).

Quant à D. mespiliformis (Tableau 2), la forêt dense présente le diamètre moyen et la surface terrière $\left(18,48 \mathrm{~cm}\right.$ et $1,80 \mathrm{~m}^{2} / \mathrm{ha}$, respectivement) les plus élevés alors que la savane arborée en présente les plus faibles valeurs $\left(9,05 \mathrm{~cm}\right.$ et $0,29 \mathrm{~m}^{2} / \mathrm{ha}$, respectivement). La forêt dense présente la contribution en surface terrière $(6,45 \%)$ la plus faible et la densité de régénération (1858,75 plants/ha) la plus élevée alors que la jachère présente la contribution en surface terrière $(61,10 \%)$ la plus élevée et la densité de régénération (193,33 plants/ha) la plus faible. La densité d'arbres la plus élevée était observée en savane arborée (44,44 tiges/ha) alors que la plus faible (22,22 tiges/ha), en jachère.

Les caractéristiques dendrométriques de $V$. doniana par formation végétale (Tableau 2) révèlent que les valeurs les plus élevées de densité $(30,21$ tiges/ha), de diamètre moyen $(16,41 \mathrm{~cm})$ et de surface terrière $\left(0,97 \mathrm{~m}^{2} / \mathrm{ha}\right)$ des arbres étaient observées en forêt dense alors que les valeurs les plus (10,32 tiges/ha, 
$13,48 \mathrm{~cm}$ et $0,76 \mathrm{~m}^{2} / \mathrm{ha}$ respectivement), en savane arborée. Toujours en savane arborée, la plus forte contribution en surface terrière $(25,09 \%)$ et la plus faible densité de régénération $(55,00$ plants/ha $)$ ont été observées alors que la plus faible contribution en surface terrière $(4,05 \%)$ a été observée en forêt dense et la plus forte densité de régénération (126,67 plants/ha), en jachère.

L'analyse des valeurs moyennes des caractéristiques dendrométriques des formations végétales (Tableau 2) révèle que la forêt dense avait une densité moyenne de 937,50 tiges/ha pour un diamètre moyen de $18,94 \mathrm{~cm}$ et une surface terrière de 25,82 $\mathrm{m}^{2} /$ ha. D. mespiliformis présentait les densités d'arbres et de régénération les plus élevées alors que A. paniculata en présentait les plus faibles valeurs. En ce qui concerne le diamètre moyen et la surface terrière moyenne, $A$. paniculata présentait les valeurs les plus élevées alors que $V$. doniana en présente les valeurs les plus faibles.

La savane arborée quant à elle, présentait une densité d'arbres de 136,51 tiges/ha pour un diamètre moyen de $18,81 \mathrm{~cm}$ et une surface terrière moyenne de $2,77 \mathrm{~m}^{2} / \mathrm{ha}$ (Tableau 2). D. mespiliformis présentait les densités moyenne et de régénération les plus élevées, le diamètre moyen et la surface terrière les plus faibles alors que $V$. doniana présentait les plus faibles valeurs de densités d'arbres et de régénérations mais les plus fortes valeurs de diamètre moyen et de surface terrière. Quant à A. paniculata et K. africana, elles sont absentes en savane arborée (en terme d'individus adultes).

En ce qui concerne la jachère, elle présentait une densité moyenne, un diamètre moyen et une surface terrière de l'ordre de 60 tiges/ha, $17 \mathrm{~cm}$ et $2 \mathrm{~m}^{2} / \mathrm{ha}$ respectivement (Tableau 2). Des quatre espèces cibles, seule D. mespiliformis a été observée dans la jachère (Tableau 2).

\section{Structures en diamètre}

Les structures en diamètre établies pour la forêt dense et la savane arborée (Figures $3 \mathrm{~A}$ et $3 \mathrm{~B}$ ) montrent une distribution en "J renversé" $(c<1)$, caractéristique des peuplements multispécifiques ou inéquiennes. En ce qui concerne la jachère, la structure en diamètre (Figure $3 \mathrm{C}$ ) présente une distribution asymétrique droite avec un paramètre de forme de $1,378(1<\mathrm{c}<3,6)$, caractéristique des peuplements monospécifiques avec prédominance d'individus jeunes ou de faibles diamètres. La classe de diamètre $10-20 \mathrm{~cm}$ est la plus représentée dans les trois formations végétales alors que les individus de diamètre supérieur à $50 \mathrm{~cm}$ y sont rares. Les trois structures s'ajustent bien à la distribution théorique de Weibull à 3 paramètres (Prob.>0,05; Tableau 3).

La distribution en diamètre établie pour D. mespiliformis (Figure 4) indique une allure exponentielle négative, un "J renversé" et une asymétrie droite $(\mathrm{c}=1,870)$ respectivement en forêt dense, savane arborée et jachère. La distribution en diamètre établie pour $V$. doniana révèle une asymétrie droite avec un paramètre de forme variant de 1,013 (figure 5A) à 1,185 (Figure 5B). Pour A. paniculata et $K$. africana, uniquement observées en forêt dense, la première présente une structure diamétrique exponentielle négative (Figure 6A) alors que la deuxième présente une distribution asymétrique droite avec un paramètre de forme de 1,460 (Figure 6B). Pour les 4 espèces, les individus les plus représentés présentent de faible diamètre (inférieur ou égal à $20 \mathrm{~cm}$ ). Les individus de diamètre supérieur à $50 \mathrm{~cm}$ ne sont observés que pour A. paniculata, K. africana et $D$. mespiliformis en forêt dense. Par ailleurs, toutes les structures en diamètre établies pour les 4 espèces s'ajustent bien soit à la distribution exponentielle soit à la distribution de Weibull (prob. > 0,05 pour les tests d'ajustement log-linéaire ; Tableau 3).

Effets du niveau des pressions anthropiques sur les caractéristiques structurales des espèces cibles

Les corrélations entre caractéristiques structurales des espèces cibles et le niveau de pression dans les peuplements (Tableau 4) sont globalement non significatives. Seule la densité d'arbres de $V$. doniana variait significativement suivant le niveau de 
pression (Prob. $=0,000)$ et de façon surprenante la corrélation était positive $(0,666)$. Ainsi, plus le niveau de pression est élevé dans le peuplement, plus élevée y est la densité de $V$. doniana. Ceci suggère que la pression sur les peuplements serait orientée vers d'autres espèces, ce qui favorise le développement de $V$. doniana. En ce qui concerne les autres espèces, de faibles corrélations non significatives ont été notées.
Les corrélations étaient négatives entre les niveaux de pression et les densités d'une part et entre les niveaux de pression et le diamètre moyen (respectivement la surface terrière) de D. mespiliformis d'autre part. En d'autres termes, les pressions anthropiques ont négativement impacté les densités d'arbres de A. paniculata, $D$. mespiliformis et $K$. africana, mais aussi le diamètre moyen et la surface terrière de $D$. mespiliformis.

Tableau 1 : Paramètres de diversité floristique des formations végétales.

\begin{tabular}{lrrr}
\hline Paramètres de diversité & $\begin{array}{r}\text { Forêt dense } \\
(\mathbf{n = 3 2})\end{array}$ & $\begin{array}{r}\text { Savane arborée } \\
(\mathbf{n = 1 4})\end{array}$ & $\begin{array}{r}\text { Jachère } \\
(\mathbf{n = 1 4})\end{array}$ \\
\hline Richesse spécifique globale $(S$, espèces $)$ & 92,00 & 6,00 & 5,00 \\
Diversité de Shannon $(H$, bits $)$ & 4,86 & 1,55 & 2,11 \\
Equitabilité de Pielou, $E q$ & 0,74 & 0,60 & 0,91 \\
\hline
\end{tabular}

Indice de valeur d'importance

(IVI)

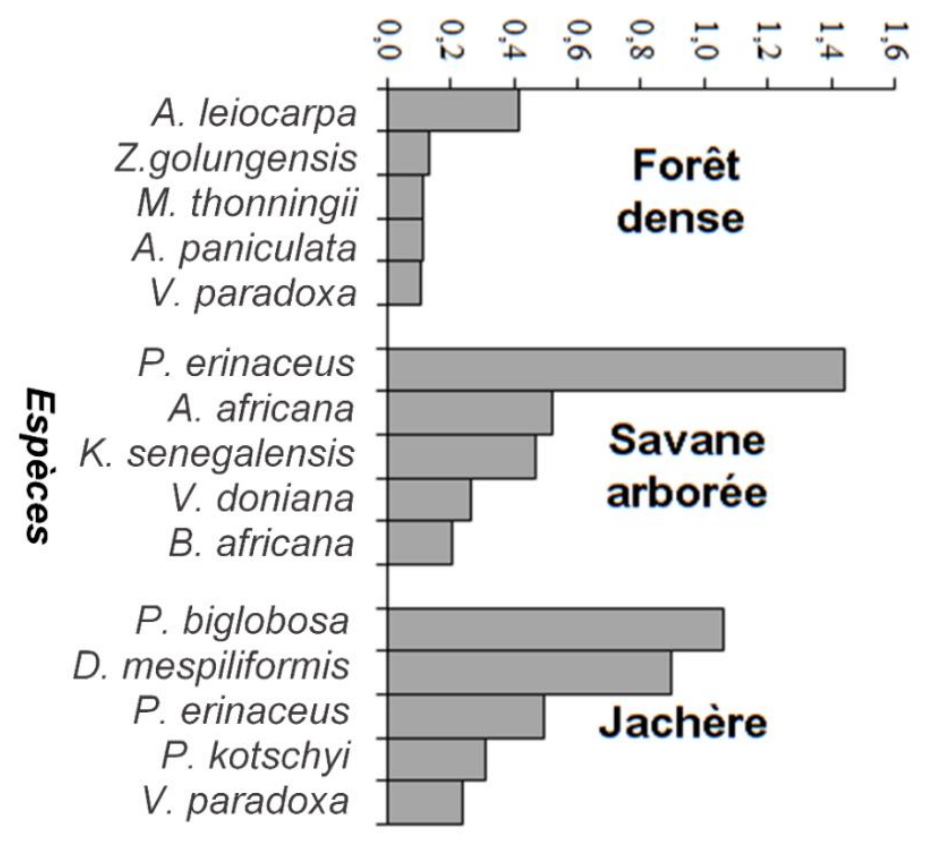

Figure 2 : Principales espèces caractéristiques des formations végétales identifiées. 
Tableau 2 : Caractéristiques dendrométriques des formations végétales.

\begin{tabular}{|c|c|c|c|c|c|c|c|c|}
\hline \multirow[t]{2}{*}{ Paramètres } & & \multicolumn{2}{|c|}{$\begin{array}{c}\text { Forêt dense } \\
(n=32)\end{array}$} & \multicolumn{2}{|c|}{$\begin{array}{c}\text { Savane } \\
\text { arborée }(n=14)\end{array}$} & \multicolumn{2}{|c|}{$\begin{array}{c}\text { Jachère } \\
(n=14)\end{array}$} & \multirow[t]{2}{*}{ Prob. } \\
\hline & & $m$ & $C v(\%)$ & $m$ & $C v(\%)$ & $m$ & $C v(\%)$ & \\
\hline \multirow{5}{*}{$\begin{array}{l}\text { Densité } \\
(N, \text { tiges } / h a)\end{array}$} & A. paniculata & 2,43 & 397,76 & - & - & - & - & - \\
\hline & D. mespiliformis & 30,21 & 95,84 & 44,44 & - & 22,22 & 0,00 & 0,842 \\
\hline & K. africana & 20,14 & 133,27 & - & - & - & - & - \\
\hline & V. doniana & 30,21 & 165,35 & 10,32 & 317,41 & - & - & 0,004 \\
\hline & Global & 937,50 & 27,04 & 136,51 & 106,87 & 59,26 & 92,49 & $\mathbf{0 , 0 0 0}$ \\
\hline \multirow{5}{*}{$\begin{array}{l}\text { Diamètre moyen } \\
(D, c m)\end{array}$} & A. paniculata & 52,78 & 26,09 & - & - & - & - & - \\
\hline & D. mespiliformis & 18,48 & 85,93 & 9,05 & - & 14,28 & 16,58 & 0,768 \\
\hline & K. africana & 26,54 & 30,69 & - & - & - & - & - \\
\hline & $V$. doniana & 16,41 & 57,27 & 13,48 & 32,72 & - & - & 0,742 \\
\hline & Global & 18,94 & 16,43 & 18,81 & 29,96 & 16,59 & 15,98 & 0,696 \\
\hline \multirow{5}{*}{$\begin{array}{l}\text { Surface terrière } \\
\left(G, m^{2} / h a\right)\end{array}$} & A. paniculata & 8,35 & 31,88 & - & - & - & - & - \\
\hline & D. mespiliformis & 1,80 & 203,82 & 0,29 & - & 0,36 & 31,03 & 0,849 \\
\hline & K. africana & 2,31 & 77,73 & - & - & - & - & - \\
\hline & $V$. doniana & 0,97 & 109,75 & 0,76 & 51,43 & - & - & 0,704 \\
\hline & Global & 25,82 & 26,64 & 2,77 & 69,66 & 1,55 & 118,48 & $\mathbf{0 , 0 0 0}$ \\
\hline \multirow{4}{*}{$\begin{array}{l}\text { Contribution en } \\
\text { surface terrière } \\
(C s, \%)\end{array}$} & A. paniculata & 26,38 & 52,54 & - & - & - & - & - \\
\hline & D. mespiliformis & 6,45 & 180,24 & 11,01 & - & 61,10 & 79,86 & 0,015 \\
\hline & K. africana & 7,92 & 82,50 & - & - & - & - & - \\
\hline & $V$. doniana & 4,05 & 119,73 & 25,09 & 36,98 & - & - & $\mathbf{0 , 0 2 6}$ \\
\hline $\begin{array}{l}\text { Densité de } \\
\text { régénération }\end{array}$ & A. paniculata & 20,00 & - & - & - & - & - & - \\
\hline \multirow[t]{3}{*}{$(N r$, plants $/ h a)$} & D. mespiliformis & 1858,75 & 129,18 & 220,00 & 142,59 & 193,33 & 96,35 & $\mathbf{0 , 0 0 0}$ \\
\hline & K. africana & 133,33 & 108,51 & - & - & 260,00 & - & 0,528 \\
\hline & V. doniana & 58,67 & 91,54 & 55,00 & 104,45 & 126,67 & 132,42 & 0,747 \\
\hline
\end{tabular}

Différences non significatives lorsque Prob. $=5 \%$; différences significatives lorsque Prob. $=1 \%$; différences hautement significative lorsque Prob. $=0.1 \%$. Moyenne $(m)$, coefficient de variation $(C v)$ et valeur de probabilité $($ Prob.)
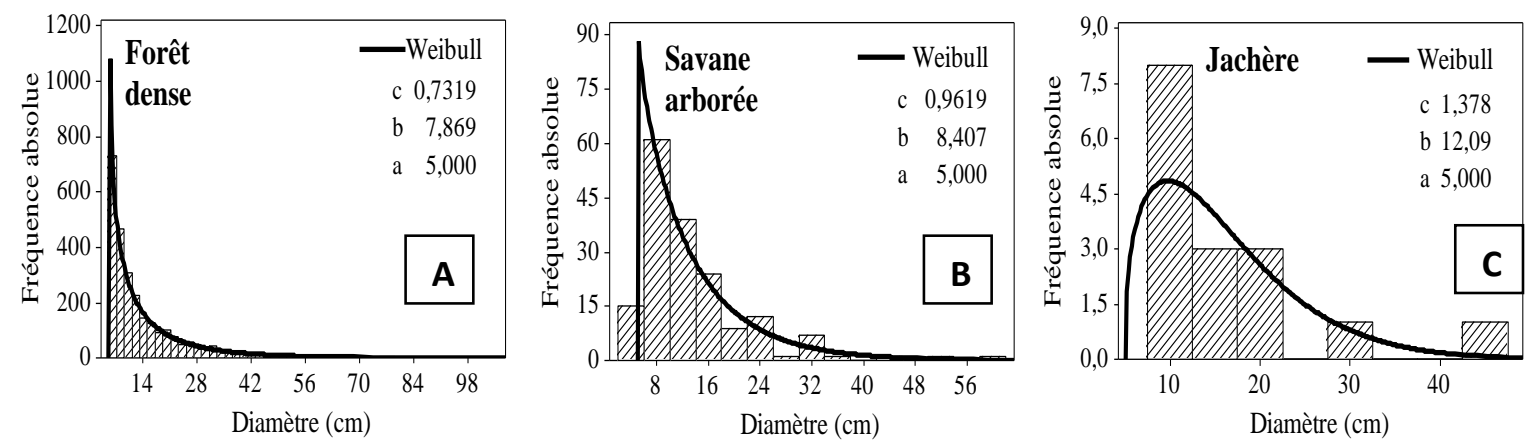

Figure 3 : Structures en diamètre des formations végétales étudiées. 
Tableau 3: Ajustement des distributions en diamètre aux distributions exponentielle et de Weibull : résultats d'analyse log-linéaire.

\begin{tabular}{|c|c|c|c|c|c|c|}
\hline \multirow{2}{*}{ Populations } & \multicolumn{2}{|c|}{ Forêt dense $(n=32)$} & \multicolumn{2}{|c|}{ Savane arborée $(n=14)$} & \multicolumn{2}{|c|}{ Jachère $(n=14)$} \\
\hline & Chi 2 & Prob. & Chi 2 & Prob. & Chi 2 & Prob. \\
\hline A. paniculata & 1,59 & 0,208 & - & - & - & - \\
\hline D. mespiliformis & 0,78 & 0,376 & 2,66 & 0,103 & 0,06 & 0,800 \\
\hline K. africana & 0,82 & 0,365 & - & - & - & - \\
\hline V. doniana & 0,20 & 0,655 & 0,92 & 0,338 & - & - \\
\hline Global & 1,59 & 0,207 & 0,25 & 0,616 & 0,75 & 0,385 \\
\hline
\end{tabular}

Différences non significatives lorsque Prob. $=5 \%$; différences significatives lorsque Prob. $=1 \%$; différences hautement significative lorsque Prob. $=0.1 \%$.
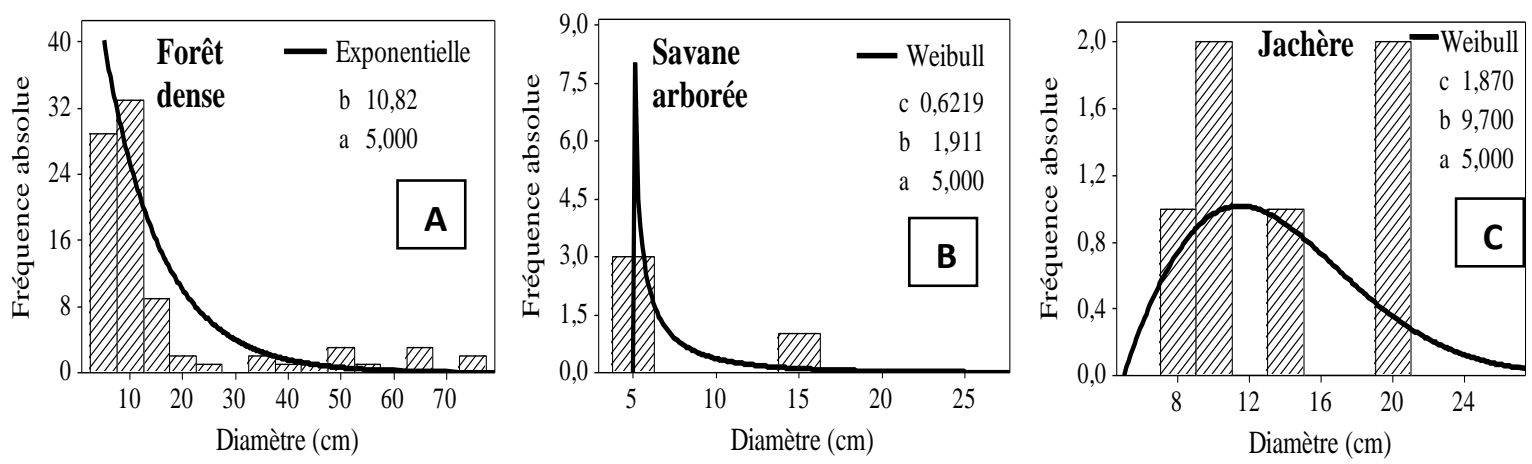

Figure 4 : Structures en diamètre de D. mespiliformis dans les trois formations végétales.
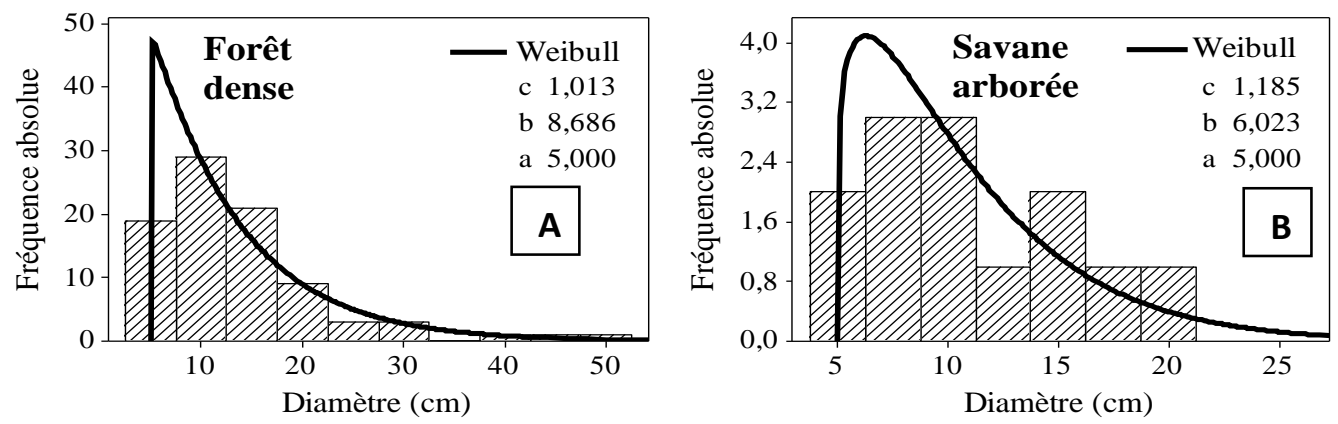

Figure 5 : Structures en diamètre de V. doniana en forêt dense et savane arborée
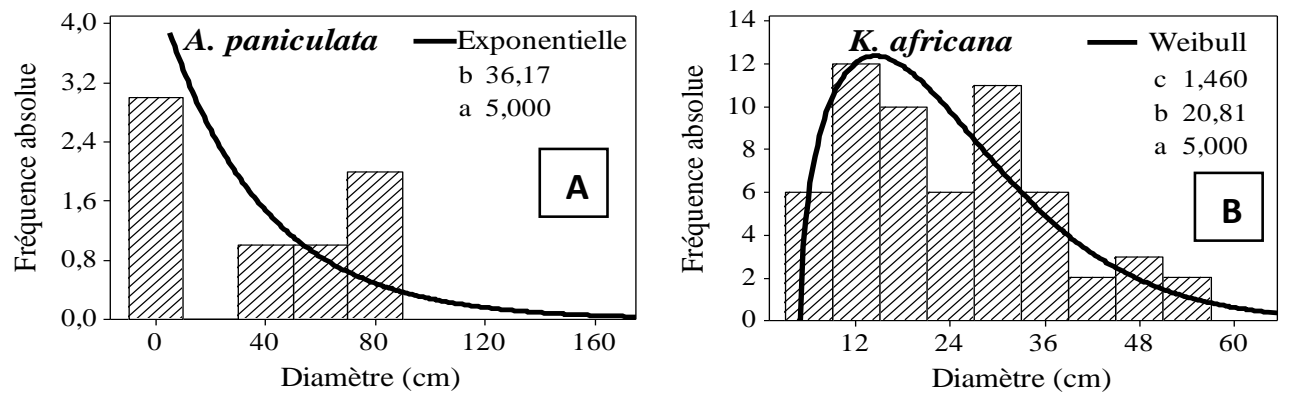

Figure 6 : Structures en diamètre de A. paniculata et $K$. africana dans la forêt dense. 
Tableau 4: Corrélations entre le niveau de pressions sur les populations et les paramètres dendrométriques: corrélation de Spearman et probabilité de signification (en italique).

\begin{tabular}{lrrrr}
\hline Espèces & Densité $(\mathbf{N})$ & Diamètre (D) & Surface terrière (G) & Régénérations (Nr) \\
\hline A. paniculata & $-0,347$ & - & - & - \\
& 0,052 & - & - & - \\
D. mespiliformis & $-0,032$ & $-0,243$ & $-0,356$ & 0,172 \\
& 0,856 & 0,222 & 0,068 & 0,229 \\
K. africana & $-0,075$ & 0,196 & 0,140 & 0,738 \\
& 0,683 & 0,467 & 0,605 & 0,262 \\
V. doniana & 0,666 & 0,051 & 0,214 & 0,103 \\
& $\mathbf{0 , 0 0 0}$ & 0,817 & 0,340 & 0,650 \\
\hline
\end{tabular}

Différences non significatives lorsque Prob. $=5 \%$; différences significatives lorsque Prob. $=1 \%$; différences hautement significative lorsque Prob. $=0.1 \%$.

\section{DISCUSSION}

\section{Caractéristiques écologique des formations végétales}

La présente étude a permis de décrire la structure forestière de trois principaux types d'habitats hébergeant les espèces-cibles à savoir Afraegle paniculata, Diospyros mespiliformis, Kigelia africana et Vitex doniana. En effet, la non individualisation de chaque type de formation végétale de base peut être expliquée par une homogénéité relative observée dans la composition floristique au sein de chaque formation végétale. La diversité spécifique élevée dans la forêt dense révèle l'équiprobabilité de différentes espèces et montre l'importance de ce type d'habitat dans la conservation de la diversité biologique au sein de la forêt sacrée de Nassou. C'est pourquoi, en Afrique, en Amérique latine et en Asie, les sites sacrés, en particulier les forêts sacrées, intéressent de plus en plus les scientifiques et les organismes de protection de la nature (Swamy et al., 2003). Dans les pays à faible couverture végétale tels que le Bénin, l'intérêt des forêts sacrées dans le maintien des reliques forestières anciennes et la conservation de la biodiversité n'est plus à démontrer. Ces forêts sacrées sont de véritables réservoirs de la diversité biologique (Toko et al., 2015; Agbani et al., 2017).
Dans les formations végétales considérées, en dehors de D. mespiliformis qui est reconnue écologiquement importante dans la jachère, aucune autre espèce-cible n'est reconnue ni écologiquement faible, ni écologiquement importante. Cela voudrait signifier que les espèces-cibles contribuent moyennement dans la valeur écologique de leurs habitats respectifs. L'importance écologique accordée à $D$. mespiliformis dans la jachère serait due au fait que certains pieds de cet arbre sont souvent laissés dans les systèmes agricoles à cause de sa sacralisation et leur utilisation en bois d'œuvre. $D$. mespiliformis est une espèce reconnue comme abritant des esprits bienfaisants et facilite la communication avec le génie de la forêt et surtout des termitières. Son gui est d'ailleurs particulièrement recherché pour la réalisation des produits de la chance et la prospérité matérielle et financière. Il est de coutume reconnu dans l'ethnie peulh qu'un individu de $D$ mespiliformis portant un gui est systématiquement émondé ou abattu comme c'est le cas aussi avec Annona senegalensis afin de bénéficier pleinement des biens que procurerait cette trouvaille qui est assez rare. Pour ce qui est des autres espèces-cibles, leur faible pouvoir de régénération peut expliquer aussi leur faible représentativité au sein des différentes formations végétales. Chacune de ces espèces ont une importance socio- 
culturelle auprès des populations locales d'où leur maintien au sein de la forêt de Nassou.

En savane arborée et en jachère, on note une faible diversité biologique liée à la dominance de certaines espèces telles que $V$. paradoxa, $P$. erinaceus, $P$. kotshyii. Par ailleurs, la faible diversité des ligneux observée au niveau des jachères n'est qu'une conséquence de la pression anthropique.

\section{Caractéristiques dendrométriques}

Les résultats de l'étude ont montré que quelle que soit l'espèce cible considérée, la densité de régénération est supérieure à celle des pieds adultes (Tableau 2). Cela signifie que les graines de chacune de ces plantes sont bien disséminées et germent facilement. Malgré la densité élevée des plantules comparativement aux pieds adultes, au niveau de A. paniculata (20 pieds/ha) et de $V$. doniana (58 pieds/ha), la valeur calculée est un peu faible. Ces résultats montrent que les populations des espèces considérées se renouvellent normalement mais avec un faible pouvoir de reprise et de survie. En ce qui concerne les plantes qui sont retrouvées dans plusieurs habitats à la fois, le mode de recrutement des plantules n'est pas le même. Au niveau de D. mespiliformis, on constate que la densité de régénération diminue suivant le gradient de végétation forêt dense > savane $>$ jachère. La faible densité de régénération de D. mespiliformis en jachère et en savane peut être expliquée par les feux de végétation qui déciment les semences et certaines plantules plus vulnérables qui n'arrivent pas à y résister et, par l'élagage des sujets adultes qui agit sur la fructification ou par certains paramètres abiotiques du milieu tels que la nature du sol (facteurs pédologique), l'humidité et l'ensoleillement. Cette variation de densité des plantules de D. mespiliformis ne suit pas la même logique au niveau des pieds adultes où on observe la plus forte densité dans la savane arborée et la plus faible en jachère. Cela démontre de plus que le fort taux de régénération n'induit pas forcément le plus grand nombre de pieds adultes. Ce cas spécifique confirme le type d'habitat pourrait amener à dire qu'en forêt dense, cette plante se développe mieux, fructifie et germe plus qu'en savane et en jachère. De plus, la densité de régénération élevé des jeunes plantes pourrait induire un potentiel de résilience à la forêt de Nassou comme l'on montré les travaux de Hounkpevi et al. (2011). Cependant, pour mieux apprécier la dynamique de population de chacune de ces espèces, une étude de suivi de la croissance de chacune de ces plantes est nécessaire quand bien même cela doit prendre plusieurs années.

Sur le plan structural, les individus jeunes ou de faible diamètre sont majoritaires avec un recrutement régulier au niveau de la forêt dense. Ceci témoigne la reconstitution de ces formations végétales en absence d'une forte pression anthropique. Ces résultats rejoignent ceux de Assogbadjo et al. (2009) dans la forêt classée de Wari-Maro au centre du Bénin, Bonou et al. (2009) dans la forêt classée de la Lama au Sud du Bénin et de Assongba et al. (2013) au Sud-est du pays. Pour ces types d'habitats, les tiges d'avenir sont en nombre suffisamment élevé et le renouvellement des peuplements est assuré. Un tel habitat contient des espèces dites structurantes car assurant leur pérennité dans les peuplements (Sokpon et Biaou, 2002). Différentes raisons peuvent expliquer cette prédominance des individus à petit diamètre. Elle peut être liée au fait que la stratégie de régénération dominante des espèces dans ces types d'habitats soit le drageonnement et que les jeunes individus restent longtemps dépendants des pieds mères avant de s'affranchir dès qu'une ouverture suffisante se fait dans la végétation. Dans ce cas, les résultats de la présente étude sont conformes à l'idée développée par Fandohan et al. (2010) lors de leur recherche sur la régénération de Tamarindus indica au Bénin. En outre, les individus à grand diamètre pourraient être régulièrement détruits pour des besoins d'ordre socio-économique. Au niveau de la jachère, les résultats issus de l'ajustement à la distribution de Weibull montrent clairement qu'il s'agit d'un habitat où la prédominance d'une espèce ou d'un groupe restreint d'espèces est notée ; ce qui est en adéquation aux caractéristiques écologiques d'une zone fortement perturbée.

En considérant la structure diamétrique ajustée à Weibull de chacune des espèces 
étudiées, le cas de A. paniculata qui n'est retrouvée que dans la forêt dense ne présente pas une structure régulière. $\mathrm{Ce}$ résultat renforce la liste rouge sur le Bénin, élaborée pour connaître le statut de conservation des espèces végétales et animales (Neuenschwender et al., 2011) A. paniculata est une espèce qui subit une forte pression anthropique sur tous les organes en particulier les racines, les écorces du tronc et les fruits. En effet les racines sont systématiquement récoltées pour la bonne santé des femmes après les accouchements. Sa raréfaction a entraîné la ruée sur les racines de Zanthoxylum zanthoxyloides. De nos jours, la raréfaction de cette dernière espèce aussi prisée pour les mêmes causes à fait porter le choix sur les citrus de la même famille des Rutaceae. Des études complémentaires de pharmacognosie permettront de meilleures valorisations des divers usages médicinaux des organes de ces espèces.

L'analyse des impacts probable des pressions anthropiques sur les paramètres dendrométriques des espèces-cibles révèle une corrélation négative quoique globalement non significative. Cela suggère que la pression anthropique est portée vers d'autres espèces à valeur d'usage élevé. La faible densité obtenue en milieu perturbé comparativement au milieu peu perturbée s'explique par le système de culture itinérante sur brûlis. Sinsin et al. (2015) et Dicko et al. (2016) ont montré l'effet des feux sur la densité des plantules et sur la croissance des arbres. Le choix sélectif de certains individus des espèces pour leur fruit ou leur écorce affecte la structure en diamètre qui présente des absences de classes (Figures 4, 5 et 6 ) Les pressions portées sur les individus mâtures affecteraient leur potentialité de floraison, de fructification et de régénération et limite ainsi la viabilité des espèces (Dicko et al. 2016).

\section{Conclusion}

La présente étude a permis de faire la caractérisation structurale de la forêt sacrée de Nassou, qui demeure à l'instar des formations végétales de même type un réservoir de conservation de la phytodiversité. Les résultats ont révélé que la forêt se trouve toujours dans une dynamique évolutive mais subit quelque pression sélective notamment par la recherche de produits forestiers non ligneux d'importance. Cela signifie donc que des stratégies de conservation doivent être plus orientées vers des groupes d'espècescibles pour assurer la conservation de la diversité biologique de cette forêt.

\section{CONFLIT D'INTERETS}

Les auteurs déclarent qu'il n'ont aucun conflit d'intérêts.

\section{CONTRIBUTIONS DES AUTEURS}

PA a réalisé les travaux sous les avis et la supervision de FA et BS. AA a analysé les données sous la supervision de FA. PA, AA et GC ont rédigé le manuscrit qui a été amélioré par BS. Tous les auteurs ont lu et approuvé le manuscrit.

\section{REMERCIEMENTS}

Les auteurs remercient les populations locales qui ont activement participé à cette étude.

\section{REFERENCES}

Achigan Dako EG, Pasquini MW, Assogba Komlan F, N'danikou S, Yédomonhan H, Dansi A, Ambrose Oji B. 2010. Traditional vegetables in Benin. Institut National des Recherches Agricoles du Bénin. Imprimeries du CENAP : Cotonou.

Agbani OP, Gandji K, Tovissodé F, Karen H, Sinsin B. 2017. Production fruitière de quatre essences ligneuses dans la forêt de Nassou en zone soudanienne du Bénin. European Scientific Journal, 13(36): 352-367. Doi: 10.19044/esj.2017.v13n36p352.

Agbessi KGE, Ouedraogo M, Camara M, Segniagbeto H, Houngbedji MB, Kabre AT. 2017. Distribution spatiale du singe à ventre rouge, Cercopithecus erythrogaster erythrogaster Gray et les menaces pesant sur sa conservation durable au Togo. International Journal of Biological and Chemical Sciences, 11(1): 157-173. DOI: http://dx.doi.org/10.4314/ijbcs.v11i1.13.

Ali KFMR, Odjoubere J, Tenté ABH, Sinsin B. 2014. Caractérisation floristique et 
analyse des formes de pression sur les forêts sacrées ou communautaires de Basse Vallée de l'Ouémé au Sud-Est du Bénin. Afrique Science, 10(2): 243-257. http://www.afriquescience.info/documen t.php?id=3458.

Alohou EC, Gbemavo DSJC, Ouinsavi C, Sokpon N. 2016. Local perceptions and importance of endogenous beliefs on sacred groves conservation in South Benin. International Journal of Biodiversity and Conservation, 8(5): 105-112. DOI: 10.5897/IJBC2015.0918

Assogbadjo AE, Glèlè Kakaï RL, Sinsin B, Dieter P. 2009. Structure of Anogeissus leiocarpa Guill., Perr. natural stands in relation to anthropogenic pressure within Wari-Maro forest reserve in Benin. African Journal of Ecology, 48(3): 644-653. DOI: $10.1111 / \mathrm{j} .1365-$ 2028.2009.01160.x.

Assongba YF, Djègo GJ, Sinsin B. 2013. Distribution des habitats de Dialium guineense willd (Fabaceae: Caesalpinioideae) dans les phytodistricts Est du Sud-Bénin. Bulletin scientifique de l'Institut national pour l'environnement et la conservation de la nature, 12: 1-16.

Bonou W, Glèlè KR, Assogbadjo AE, Fonton HN, Sinsin B. 2009. Characterisation of Afzelia africana $\mathrm{Sm}$. habitat in the Lama forest reserve of Benin. Forest Ecology and Management, 258: 1084-1092. DOI:10.1016/j.foreco.2009.05.032

Cardelús CL, Scull P, Hair J, Baimas-George M, Lowman MD, Wassie Eshete A. 2013. A preliminary assessment of Ethiopian sacred grove status at the landscape and ecosystem scales. Diversity, 5(2): 320-334. DOI: 10.3390/d5020320.

Curtis JT, McIntosh RP. 1951. An upland forest continuum in the prairie forest border region of Wisconsin. Ecology, 32(3): 476-496. http://www.jstor.org/stable/1931725.

Dicko A, Biaou SSH, Natta AH, Gado CAS, Kouagou M. 2016. Influence des pressions anthropiques sur la structure des populations de Pentadesma butyracea au Bénin. VertigO - la revue électronique en sciences de l'environnement, 16(3) | décembre 2016. DOI : 10.4000/vertigo.18195

Dudley N, Higgins-Zogib L, Mansourian S. 2009. The links between protected areas, faiths, and sacred natural sites. Conservation Biology, 23(3): 568-577. DOI: $10.1111 /$ j.15231739.2009.01201.x

Fandohan $\mathrm{AB}$, Assogbadjo AE, Glèlè Kakaï RL, Sinsin B, Van Damme P. 2010. Impact of habitat type on the conservation status of tamarind (Tamarindus indica L.) populations in the W National Park of Benin. Fruits, 65(1): $\quad 11-19$. 10.1051/fruits/2009037.

Gutiérrez OS. 2014. Protection of the sacred lands and culture of the Wixárika people. Domestic and international legal perspective over the Wixárika mining case. Master thesis, University of Lapland, 134p.

Hounkpèvi A, Yêvidé ASI, Ganglo JC, Devineau J-L, Azontonde AH, Adjakidje V, Agbossou EK, De Foucault B. 2011. Structure et écologie de la forêt à Diospyros mespiliformis Hochst. ex A.DC. et à Dialium guineense Willd. de la réserve de Massi (La Lama), Bénin. Bois et Forêts des Tropiques, 308(2): 33-46. DOI: https://doi.org/10.19182/bft2011.308.a2 0472

Johnson NL, Kotz S. 1970. Distributions in Statistics: Continuous Univariate Distributions. New York: John Wiley \& Sons.

Juhé-Beaulaton D. 2010. Forêts sacrées et sanctuaires boisés. Des créations culturelles et biologiques (Burkina Faso, Togo, Bénin). France. Karthala, pp.280.

Kangas A, Maltamo M. 2007. Forest Inventory: Methodology and Applications. Dordrecht (England): Springer.

Kokou K, Sokpon N. 2006. Les forêts sacrées du couloir de Dahomey. Bois et Forêts des Tropiques, 288(2): 15-23. DOI: https://doi.org/10.19182/bft2006.288.a2 0312. 
Neuenschwander $\mathrm{P}$, Bown $\mathrm{D}$, Hèdégbètan GC, Adomou A. 2015. Long-term conservation and rehabilitation of threatened rain forest patches under different human population pressures in West Africa. Nature Conservation, 13: 21-46.

DOI:

10.3897/natureconservation.13.6539

Neuenschwander P, Sinsin B, Georgen G. 2011. Protection de la Nature en Afrique de l'Ouest : Une Liste Rouge pour le Bénin. International Institute of Tropical Agriculture, Ibadan, Nigéria. 365 p.

Ormsby AA, Bhagwat SA. 2010. Sacred forests of India: a strong tradition of community-based natural resource management. Environmental Conservation, 37(3): $\quad 320-326$. https://doi.org/10.1017/S037689291000 0561

R Core Team. 2013. R: A language and environment for statistical computing. $\mathrm{R}$ Foundation for Statistical Computing, Vienna, Austria. URL http://www.Rproject.org/.

Rondeux J. 1999. La mesure des peuplements forestiers. 2èmeeds.

Gembloux (Belgique):

Presses agronomiques de Gembloux.

Savadogo S, Kabore A, Thiombiano A. 2017. Caractéristiques végétales, typologie et fonctions des bois sacrés au Burkina Faso. Int. J. Biol. Chem. Sci., 11(4): 1497-1511.

DOI: http://dx.doi.org/10.4314/ijbcs.v11i4.8

Siebert U, Elwert G. 2004. Combating corruption and illegal logging in Bénin, West Africa, Journal of Sustainable Forestry, 19: (1-3), 239-261. DOI: https://doi.org/10.1300/J091v19n01_11

Sinsin F, Glèlè Kakaï R, Orthmann B, Sinsin B. 2015. Tree-ring: a suitable implement for spatial and temporal fire distribution analysis in savanna woodland and dry forest. Journal of Forestry research, 26(2); 435-446. DOI: 10.1007/s11676015-0047-7.
Sokpon N, Biaou G. 2002. The use of diameter distributions in sustained-use management of remnant forests in Benin: case of Bassila forest reserve in North Benin. Forest Ecology and Management, 161(1-3): 13-25. https://doi.org/10.1016/S0378-1127 (01)00488-1

Swamy PS, Kumar M, Sundarapandian SM. 2003. Spirituality and ecology of sacred groves in Tamil Nadu, India. Unasylva, 213(54): 53-58.

Tamalene MM, Al Muhdhar MHI, Suarsini E, Rochman F. 2014. The practice of local wisdom of Tobelo Dalma (Togutil) Tribal community in forest conservation in Halmahera, Indonesia. International Journal of Plant Research, 4 (4A): 1-7. DOI:10.5923/s.plant.201401.01

Toko Imorou I, Djogbenou PC, Arouna O, Sogbossi Sogbohossou E, Sinsin B. 2015. Effets de la taille et des régions phytogéographiques sur la diversité floristique et la structure des forêts sacrées au Bénin. Annales des Sciences Agronomiques, 19(1): 77-95. https://www.ajol.info/index.php/asab/art icle/view/145332

Udeagha AU, Udofia SI, Jacob DE. 2013. Cultural and socio-economic perspectives of the conservation of Asanting Ibiono sacred forests in Akwa Ibom State, Nigeria. International Journal of Biodiversity and Conservation, $\quad \mathbf{5}(11)$ : $\quad 696-703$. https://doi.org/10.5897/IJBC2013.0580

Yedomonhan H, Adomou AC, Aguessy M, Bossou FG. 2017. Evaluation des caractéristiques ethnobotaniques et structurales de Nesogordonia kabingaensis (K. Schum.) Capuron ex R. Germ. (Sterculiaceae) dans la forêt sacrée d'Ewè au Bénin en vue de la définition des stratégies de sa conservation. Int. J. Biol. Chem. Sci., 11(5): 2481-2494. DOI: https://dx.doi.org/10.4314/ijbcs.v11i5.42 